Mens

revue d'histoire intellectuelle de l'Amérique française

MENS

\title{
Le complexe éditorial des Jésuites et le contrôle de la lecture au Québec (1930-1960)
}

\section{Simone Vannucci}

Volume 5, numéro 2, printemps 2005

L'histoire du livre au Québec, de la Nouvelle-France au XX ${ }^{\mathrm{e}}$ siècle

URI : https://id.erudit.org/iderudit/1024361ar

DOI : https://doi.org/10.7202/1024361ar

Aller au sommaire du numéro

Éditeur(s)

Centre de recherche en civilisation canadienne-française

ISSN

1492-8647 (imprimé)

1927-9299 (numérique)

Découvrir la revue

Citer cet article

Vannucci, S. (2005). Le complexe éditorial des Jésuites et le contrôle de la lecture au Québec (1930-1960). Mens, 5(2), 431-463.

https://doi.org/10.7202/1024361ar

\section{Résumé de l'article}

La production éditoriale des communautés religieuses en fait des joueurs importants de l'histoire de l'édition au Québec. Au début du XX ${ }^{\mathrm{e}}$ siècle, les revues et annales pieuses du style Almanach de Saint-François, Messager du Sacré-Cœur ou Bulletin paroissial prospèrent et sont souvent les seules lectures familiales. La Compagnie de Jésus, dès le XIX ${ }^{\mathrm{e}}$ siècle, déploie à Montréal un appareil de propagande efficace. Au début du XX ${ }^{\mathrm{e}}$ siècle, elle rassemble sous un même toit, celui du scolasticat jésuite de l'Immaculée Conception, l'Imprimerie du Messager, la Maison des écrivains et les bureaux de ses divers apostolats. À partir de ce réseau, la communauté s'investit dans la vie sociale et intellectuelle de Montréal et joue un rôle dans le domaine éditorial, avec la parution de multiples périodiques, brochures et tracts qui font la promotion de la pensée ignatienne. L'ensemble des publications jésuites, aux objectifs souvent très différents, présente un point commun, le contrôle de la lecture et de la réception littéraire au moyen de recensions de livres et d'articles sur la littérature. 


\title{
LE COMPLEXE ÉDITORIAL DES JÉSUITES ET LE CONTRÔLE DE LA LECTURE AU QUÉBEC (1930-1960)
}

\author{
Simone Vannucci \\ Département des lettres et communications \\ Université de Sherbrooke \\ simone.vannucci@usherbrooke.ca
}

\section{Résumé}

La production éditoriale des communautés religieuses en fait des joueurs importants de l'histoire de l'édition au Québec. Au début du $\mathrm{XX}^{\mathrm{e}}$ siècle, les revues et annales pieuses du style Almanach de SaintFrançois, Messager du Sacré-Caur ou Bulletin paroissial prospèrent et sont souvent les seules lectures familiales. La Compagnie de Jésus, dès le $\mathrm{XIX}^{\mathrm{e}}$ siècle, déploie à Montréal un appareil de propagande efficace. $\mathrm{Au}$ début du $\mathrm{XX}^{\mathrm{e}}$ siècle, elle rassemble sous un même toit, celui du scolasticat jésuite de l'Immaculée Conception, l'Imprimerie du Messager, la Maison des écrivains et les bureaux de ses divers apostolats. À partir de ce réseau, la communauté s'investit dans la vie sociale et intellectuelle de Montréal et joue un rôle dans le domaine éditorial, avec la parution de multiples périodiques, brochures et tracts qui font la promotion de la pensée ignatienne. L'ensemble des publications jésuites, aux objectifs souvent très différents, présente un point commun, le contrôle de la lecture et de la réception littéraire au moyen de recensions de livres et d'articles sur la littérature.

\section{Abstract}

In the early twentieth century, Quebec's religious congregations were major players in the province's publishing industry. Parish newsletters and devotional magazines such as the Almanach de Saint-François and the Messager du Sacré- 
Cour were in their heyday and were often the only reading material available to many families. For several decades, the Company of Jesus was at the forefront of Quebec's religious publishing. It housed its various publishing endeavours under one roof in Montreal's scolasticat jésuite de l'Immaculée Conception, including the Imprimerie du Messager and the Maison des écrivains. Through their publishing network, the Jesuits played a key role in Montreal's social and intellectual life and actively promoted Ignatian thought. And though their numerous publications sought to achieve a variety of goals, they all contained book reviews and articles on literature designed to exert influence over Quebec's reading choices.

Cet article, issu de notre thèse de doctorat en études françaises intitulée Rôle structurant des actions de la Compagnie de Jésus sur la littérature et l'édition au Québec, 1930-1960, met en relief une des actions structurantes de la Société de Jésus : son influence sur la lecture, le livre et par extension sur la littérature. Pour donner une vision de l'importance éditoriale et de l'influence intellectuelle de la communauté, autant sur les élites que sur la population en général, nous avons pris en considération plusieurs des chroniques de recensions de ses plus importants organes de presse, après avoir mis en évidence le maillage serré formé par les divers outils qui servent à développer son influence.

\section{$* * *$}

Défenseurs de l'orthodoxie catholique romaine et instigateurs de la contre-réforme en Europe, les Jésuites ont apporté très tôt en Nouvelle-France leurs traits et marques spécifiques. Interdite de recrutement après la conquête anglaise, la communauté s'éteint en 1800 avec la mort du dernier jésuite de Nouvelle-France. Elle ne reviendra au Québec qu'en 1842 pour y redevenir l'éducatrice de l'élite. Depuis, on ne compte plus les personnalités qui ont été formées selon les principes et fondements des Exercices spirituels et qui ont joué 
et jouent encore un rôle important dans la société québécoise. Dans le domaine politique, Pierre Trudeau est connu pour être un ancien du Collège Jean-de-Brébeuf. De son côté, le scientifique Hubert Reeves a étudié au collège Sainte-Marie. Pour ce qui est des arts et des lettres, nous avons l'embarras du choix : Émile Nelligan, Saint-Denys Garneau, Robert Charbonneau, François Hertel, l'éditeur Claude Hurtubise, entre autres, ont aussi fréquenté ce dernier collège qui, à lui seul, suffit à établir la réputation d'éducatrice de l'élite de la Société de Jésus.

Les historiens Jean Hamelin et Nicole Gagnon, dans leur Histoire du catholicisme québécois ${ }^{1}$, constatent que les communautés religieuses ont une personnalité définie par des caractéristiques historiques, sociales et intellectuelles qui déterminent en partie leur sphère d'influence et leur place dans la société. La modestie franciscaine, écrivent-ils, contraste avec la force conquérante des Jésuites, qu'ils qualifient d'enseignants réputés, spécialistes de la formation de l'élite et experts à maîtriser la propagande, une maîtrise qui leur permet de marquer l'ensemble social de l'empreinte ignatienne, laquelle, selon Jean Lacouture, dans Jésuites, est une "tradition culturelle entée sur le classicisme, la mesure et l'équité ${ }^{2} »$.

L'article "La presse catholique », publié dans le Messager canadien en septembre 1935 par Alexandre Dugré, s.j., illustre l'intérêt que la communauté jésuite, tout comme le pouvoir catholique en général, accorde à l'imprimé, notamment à la presse, comme outil de propagande : "On a tout dit sur l'influence des journaux [écrit-il]. Inutile de répéter que les hommes d'aujourd'hui ont un cerveau de papier, que si saint Paul revenait sur terre il se ferait journaliste et que les gazettes mènent le monde ${ }^{3}$. " Pour Alexandre Dugré, les enseignants des collèges catholiques, et surtout jésuites qui éduquent l'élite de la société, doivent nécessairement faire com- 


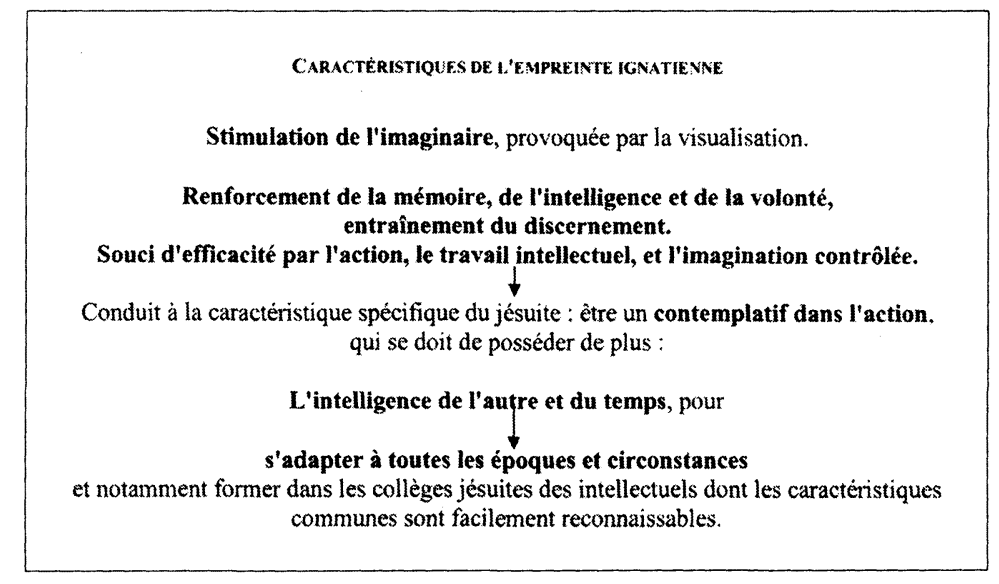

Figure 1

prendre aux jeunes gens en formation que la parole est la première forme d'apostolat, qu'il faut donc qu'ils se préparent à parler et à écrire pour servir efficacement leur religion. Ces enseignants, qui eux-mêmes prennent la plume dans les nombreux ouvrages, revues, tracts et autres imprimés que les Jésuites publient, laissent chez leurs élèves une empreinte ignatienne que l'on peut définir selon les caractéristiques présentées dans la Figure 1.

Pour implanter la pensée ignatienne dans l'ensemble social, la Société de Jésus utilise, outre sa tradition éducatrice, une grande force de persuasion que soutiennent des outils pertinents, originaux et diversifiés, essentiellement éditoriaux, qui lui permettent d'atteindre chaque élément de la société. À cette fin, elle édifie un complexe éditorial situé au scolasticat de l'Immaculée Conception à Montréal qui est un bel exemple d'efficacité. On y retrouve notamment les outils physiques et intellectuels destinés à produire toutes sortes de journaux, revues, brochures et autres imprimés qui permet- 


\section{EDIFICATION}

SCHEMA DE L'APPARELL DE PROPAGANDE

Édification et résultats

\section{PAROISSE DE L'IMMACULEE CONCEPTION}

COLASTICAT DE L TMMACUlée CONCEPTION

Faculté de Philo et de Théologie

IMPRIMERIE DU MESSAGER (1901)

Agit comme maison d'édition

MAISON DES ÉCRIVAINS

(J. P. Archambault s.j., directeur 1929)

Vivier d'auteurs (Professeurs et étudiants)

A partir de 1949 Maison Bellarmin, puis Ed. Bellarmin

ÉCOLE SOCIALE POPU AIRE (1912)

CEuvre des tracts + multitude de brochures, tracts et autres opuscules de propagande.

Publications de spiritualité

Messager du Sacré-Cour (1892-1966), Recensions litteratras

Bulletin paroissial (1909) et Bulletif de Ligues du Sacré-Cour

Rayonner et Vouloir

odèle culturel portant l'empreinte ignatienne

Diffusé par des périodiques spécifiques.

Contrôle de la réception littéraire.

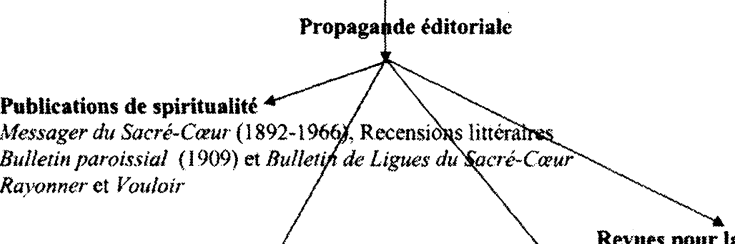

L'Actualité (1960) Articles et duroniques sur la lecture

Publications couvranme domaine social

Brochures de l'École sociale populaire

Ordre Nowveau, (oct. 1936-nov, 1940)

Relations, (1941) Recensions littéraires

Publications couvrant le domaine de l'education

Collège et famille (1944) Recensions littéraires

\section{Figure 2}


tent l'enracinement des idées jésuites. La Figure 2 présente ce complexe et ses résultats.

Dès son retour au Québec au milieu du XIX ${ }^{\mathrm{e}}$ siècle, la Société de Jésus s'investit dans de nombreux apostolats dont les sièges sociaux sont situés au collège Sainte-Marie, rue Bleury. À partir de 1907 jusqu'à 1949, la communauté les installe à son scolasticat, au Centre de l'Immaculée Conception, rue Rachel à Montréal, qui rassemble dans un même lieu les divers éléments intellectuels, propagandistes et éditoriaux propres à établir l'influence de la communauté sur la société ainsi qu'à entourer et à soutenir l'élite qu'elle forme dans ses collèges. En 1949, les directions des différents organismes apostoliques et de propagande mis en place à l'Immaculée Conception sont déplacées à la Maison Bellarmin, centre d'action religieux et social situé à l'intersection des rues SaintLaurent et Jarry, d'où se poursuivent les actions de la communauté et où, encore aujourd'hui, on retrouve les bureaux de la revue Relations ${ }^{4}$.

Outre l'église de l'Immaculée Conception dont le curé jésuite publie son Bulletin paroissial, on retrouve, rue Rachel, les facultés de philosophie et de théologie du scolasticat de la Société de Jésus, les directions des divers apostolats de la communauté, dont celui de l'« École sociale populaire ", qui diffuse la pensée sociale de la communauté dans les milieux populaires, et surtout l'Imprimerie du Messager, importante entreprise de presse qui produit les nombreuses publications jésuites à caractère religieux et social. On y retrouve aussi la direction de l'" Apostolat de la prière » qui est à l'origine des Ligues du Sacré-Cœur. Enfin, la Maison des écrivains, la Domus scriptorum $^{5}$, l'autre appareil important, sinon primordial, que l'on retrouve dans cet édifice, génère le contenu des publications, tout particulièrement les articles et les chroniques portant sur le livre et la lecture. 


\section{L'imprimerie du Messager}

L'Imprimerie du Messager, tout comme Les Relations au XVII ${ }^{e}$ siècle, a été l'outil permettant à l'appareil propagandiste de fonctionner. Elle a développé et fait rayonner les œuvres issues de l'« Apostolat de la prière ». Les Jésuites de Montréal ont su, par ce moyen, tirer profit non seulement de l'essor de l'imprimerie qui leur permettait de produire et de diffuser à bas prix une quantité importante d'imprimés, mais aussi de la scolarisation progressive de la population qui se trouvait alors plus à même d'être touchée par cette production.

Le regroupement de l'Immaculée Conception, grâce aux revues, journaux, tracts publiés par l'Imprimerie du Messager, permet à la Compagnie de Jésus de propager sa vision religieuse, politique, sociale et éducative sur tout un pan de la culture et de la société du Québec. L'appareil éditorial qu'est l'Imprimerie du Messager, créé à l'origine pour produire le Messager du Sacré-Cour, prendra une expansion telle qu'il produira au fil des ans, outre cette revue, publiée pour soutenir l'Apostolat de la prière, les Bulletins paroissiaux et les revues qui vont en découler, Action paroissiale et Ma paroisse, le Bulletin des Ligues (organe des Ligues du Sacré-Cour), le mensuel de la Ligue missionnaire étudiante, Rayonner, et celui de la Croisade eucharistique, Vouloir ${ }^{6}$. Pour l'« École sociale populaire ", l'Imprimerie publie trois séries de tracts, l'CEuvre des tracts, les Brocbures de l'"École sociale populaire ", l'Actualité en tracts $^{7}$, ainsi que les revues Relations et College et famille. Chacune des publications produites par l'Imprimerie du Messager est mise au service de l'une des nombreuses actions de la communauté et vise un but et un public très précis. En dehors des publications récurrentes, comme les revues et les journaux, le catalogue de la Bibliothèque nationale du Québec (BNQ) mentionne que de 1918 à 1980, 605 publications 
portent comme mention d'imprimeur «Imprimerie du messager » (207 livres et 358 brochures, le reste étant des tracts ou autres publications). L'imprimerie, qui agit parfois en maison d'édition (33 mentions d'éditeur de 1918 à 1980 selon la BNQ), est, comme on peut le constater, le bras actif de la propagande jésuite. Il faut souligner qu'en 1950 le personnel de l'imprimerie, qui alors faisait partie de la Maison Bellarmin, était affilié aux syndicats catholiques.

Le cas de cette imprimerie illustre bien le pragmatisme et la faculté d'adaptation qui caractérisent la spiritualité jésuite. On voit les religieux s'adapter aux technologies de l'époque et à ses avancées sociales pour assurer la médiatisation de leurs œuvres et apostolats par le biais de l'imprimé.

\section{La « Maison des écrivains »}

Afin d'assurer à leurs œuvres le rayonnement souhaité, il a fallu que les Jésuites, à l'intérieur même de leur communauté, créent un groupe affecté à alimenter en textes de toutes sortes les diverses revues, bulletins et tracts destinés à diffuser et à soutenir la propagande et les œuvres de la Société de Jésus. En 1932, on fait pour la première fois mention de « la Maison des écrivains placée sous le patronage de saint Bellarmin et affiliée à l'Immaculée Conception ${ }^{8} »$. Le même article mentionne qu'en 1942, cette « Maison » reçoit son existence canonique et autonome qui formera avec l'Imprimerie du Messager une nouvelle institution. Le Messager canadien $d u$ Sacré-Caur souligne à ce sujet que «Les écrivains et les prédicateurs de la Province des Jésuites de langue française tâchent de répandre sur un large terrain le fruit de leurs expériences ${ }^{9}$."

À propos de la "Maison des écrivains ", l'article intitulé «La Maison Bellarmin, un centre d'action religieuse et sociale ", publié par Ma paroisse en 1950, indique que le développement des œuvres «nécessita la constitution d'un groupe 
spécial à l'intérieur même de la communauté du scolasticat ${ }^{10}$ ». Sous le même toit, un ensemble de prêtres, de professeurs et d'étudiants du scolasticat produisent une grande quantité d'écrits destinés à propager la pensée ignatienne sur tous les sujets importants, religieux, politiques, sociaux et pédagogiques, qui régissent une société. Plusieurs religieux appartenant à ce groupe se sont forgé une réputation enviable dans divers domaines touchant les apostolats de la communauté. Au cours des années trente, un des premiers directeurs, le célèbre père Joseph-Papin Archambault, diffuse des textes très représentatifs du modèle culturel ignatien ${ }^{11}$ par l'intermédiaire de publications jésuites reconnues comme Le Messager du Sacré-Cour. Pierre Matte, auteur de l'article « La bibliothèque de la Maison Bellarmin " dans Livre, bibliothèque et culture québécoise. Mélanges offerts à Edmond Desrochers, s.j, explique de plus que ce groupe favorise l'éclosion d'idées neuves et d'œuvres nouvelles : la Compagnie de Jésus connaissait trop l'efficacité d'une telle synergie " pour ne pas l'utiliser au profit de ses œuvres $^{12} \gg$.

En 1949, installée dans les nouveaux locaux de la rue Jarry, la "Maison des écrivains ", capital symbolique de la communauté, deviendra maison d'édition, la Maison Bellarmin, qui publiera " une quinzaine de périodiques, des livres, des brochures et tracts de toutes sortes ${ }^{13}$ " puis, à la fin des années cinquante, les Éditions Bellarmin, spécialisées dans la théologie, la philosophie, le livre religieux et l'édition savante en général. Les pères jésuites s'investissent également dans différents organismes ou associations, comme l'«École sociale populaire », pour lesquels ils produisent un ensemble de brochures promotionnelles également pensées et diffusées par les écrivains du scolasticat. 


\section{L'«École sociale populaire "}

L'«École sociale populaire », fondée en 1912 et issue des fédérations des Ligues du Sacré-Cœur, est une autre branche importante du complexe de l'Immaculée Conception ${ }^{14}$. Organisme propagandiste, l'É.S.P. utilise en priorité l'écrit et, afin de bien circonscrire ses cibles, deux des quatre comités permanents du bureau de direction se consacrent aux publications et à la propagande ${ }^{15}$. L'organisme s'emploie, au moyen de tracts, d'articles dans les journaux et de conférences, à contrer les idées socialistes et communistes qui commencent à voir le jour dans les centres urbains et qui se développent rapidement au Québec ${ }^{16}$.

Dans un article sur le corporatisme militant publié en 1980 dans Recherches sociographiques, G.-Raymond Laliberté écrit, à propos de l'«École sociale populaire »:

que nous serions avec le corporatisme social prôné pendant près de deux décennies par l'« École sociale populaire » des Jésuites de Montréal, devant un véritable projet spécifique de structuration globale du Québec, une véritable théorie de réorganisation politique du Canada français ${ }^{17}$.

Le besoin de "Propagande sociale » de la Compagnie de Jésus est à l'origine de la création de l'« École sociale populaire » qui, selon Ma Paroisse en 1950, "[diffuse] la doctrine sociale de l'Église et la [fait] pénétrer dans les mœurs ${ }^{18} »$. Pour cela, elle utilise des publications mensuelles, des bulletins de presse hebdomadaires, des cercles d'études, des journées et sessions intensives de formation sociale et d'action corporative ainsi qu'un service de documentation et d'information, le tout administré au scolasticat. De plus, les Jésuites mettent sur pied une "trouvaille » particulièrement efficace, un service de presse, écrit G.-Raymond Laliberté, "qui joue le rôle de courtier en information sélectionnée, susceptible d'être 
reproduite telle quelle dans la plupart des grands et moyens journaux francophones du pays, aussi bien que dans des revues et des hebdomadaires de quartier ${ }^{19} »$. L'importance des publications de l'É.S.P. démontre la force propagandiste de la Société de Jésus. Il ne faut pas oublier que la revue Relations qui est encore publiée de nos jours avec un tirage de 4000 copies par mois portait à l'origine la mention d'édition de l'«École sociale populaire».

L'ensemble technique et intellectuel de l'Immaculée Conception permet à la Société de Jésus de publier diverses revues aux contenus éditoriaux souvent très divergents qui ont un point commun : leur intérêt pour le livre et la lecture. Chacune de celles-ci publie des chroniques «livres » qui s'attachent à adapter la pensée ignatienne à son lecteur type. Nous avons classé ces chroniques en deux groupes. Le premier comprend les publications à vocation spirituelle et socio-religieuse comme le Messager du Sacré-Cour dont le contenu éditorial, fait de textes à teneur religieuse et morale, s'adresse à un lectorat formé de personnes pieuses dont la spiritualité est une priorité. Ma paroisse, qui s'adresse a priori à des mères de famille qui œuvrent au cœur de la vie paroissiale, entre aussi dans ce groupe. Dans le second groupe, les revues couvrant le domaine social et l'éducation, nous retrouvons Relations, revue à teneur sociale destinée aux intellectuels formés par les collèges jésuites, et Collège et Famille qui s'intéresse à l'éducation et à la pédagogie, dont le lecteur cible est la famille de l'étudiant fréquentant ces mêmes collèges. Les importantes rubriques de recensions littéraires du Messager, de Relations et de Collège et Famille occupent dans chacune des revues un espace éditorial important alors que dans Ma paroisse, cette rubrique prend une forme qui semble plus approprié à son lectorat, celle d'un courrier du lecteur uniquement orienté sur la lecture. 


\section{Publications à vocation spirituelle et socio-religieuse}

\section{Le Messager canadien du Sacré-Caur}

Publication à vocation spirituelle, le Messager canadien du Sacré-Cour doit : "propager la spiritualité de l'Apostolat de la prière et [...] faire connaitre les amis, les œuvres et les intérêts du Cœur de Jésus à travers le monde ${ }^{20}$ ». Créé, en 1861, à Toulouse par le père Henri Ramière ${ }^{21}$, sous le nom de Messager $d u$ Caur de Jésus, la revue est importée au Québec par la communauté jésuite en 1892. Elle atteint en 1949 cinquante-huit éditions en trente-cinq langues différentes à travers le monde. Pour le seul Canada, Le Messager canadien est publié en quatre langues, soit en français au Québec, en anglais à Toronto, en cri pour les Amérindiens de l'Alberta et, à partir de 1956, en hongrois pour ceux qui ont fui la répression communiste en Hongrie en se réfugiant en Amérique du Nord ${ }^{22}$. La publication du Messager sera définitivement abandonnée en 1966 faute de lecteurs.

L'Imprimerie du Messager, à ses débuts, avait pour rôle unique la production de cette revue mensuelle de soixantequatre pages. L'édition en langue française du Messager canadien est une des revues de la communauté jésuite les plus répandues, et même si sa vocation première est de soutenir l'Apostolat de la prière, son contenu éditorial touche de nombreux autres sujets chers aux Jésuites, notamment tout ce qui de près ou de loin a rapport à l'éducation et à la lecture. Les articles destinés à prévenir et à guider l'abonné dans les dédales de la bonne lecture et ceux qui aideront les parents à choisir les livres et les revues pour leurs enfants et leurs adolescents abondent.

Une analyse de la rubrique de recension "Les livres" indique que pour la seule année 1930 les trente-trois pages de 
cette rubrique occupent $6 \%$ de l'espace éditorial annuel et recense 214 ouvrages. En 1940, cet espace est réduit à 1,5\% de l'ensemble éditorial et en 1941 à $0,7 \%$. En 1942, il revient à $1,5 \%$, pourcentage qui restera relativement stable jusqu'en 1949. Au cours des années cinquante, l'espace réservé à la rubrique augmente légèrement, pour culminer en 1960 à $3,3 \%$. Deux facteurs importants expliquent ces fluctuations : d'une part la guerre qui, à partir de 1940, coupe l'approvisionnement européen en livres et, d'autre part, la création de revues comme Relations et College et famille dont les chroniques de recensions plus étoffées rendent Le Messager à sa vocation première : la spiritualité.

Que les éditeurs soient Français, Italiens ou Canadiens, les ouvrages proposés par les recensions du Messager canadien sont essentiellement religieux, écrits par des religieux très souvent jésuites. Les biographies et les hagiographies abondent alors que les romans, récits et recueils de poésie sont très peu nombreux. Les recensions de littérature jeunesse, que l'on retrouve tout au long de la période dans cette revue, représentent sa manifestation littéraire la plus importante. Le père Joseph-Papin Archambault, au cours des années trente, écrit à ce sujet dans le Messager que "la jeunesse des deux sexes a besoin d'être protégée contre les lectures frivoles et immora$\operatorname{les}^{23} »$.

Le Messager, dans ses propositions de lecture, se présente comme une revue qui favorise la spiritualité et qui désire pousser ses lecteurs sur le chemin de la prière comme sa mission première le veut. Les recensions sont destinées à renforcer cet apostolat. Cela ne l'empêche pas, tant qu'il constitue la seule revue jésuite importante sur le marché, de traiter pour ses lecteurs de questions sociales et de morale tout autant que d'éducation ou d'histoire. Le Messager fait également la promotion des apostolats jésuites et sert avant tout à intro- 
duire dans les familles la pensée ignatienne et la vision sociale et morale de la communauté. En ce sens, on peut dire que le Messager sert les idées et la spiritualité de la Société de Jésus au Québec au cours de la période s'étendant du début des années trente jusqu’à la Révolution tranquille.

\section{Ma paroisse}

Le premier janvier 1945, l'Action paroissiale, directement issue des Bulletins paroissiaux, devient Ma paroisse, une revue mensuelle de vingt-quatre pages, de format $8,5 \times 11$ pouces. Elle est destinée, comme l'écrit son directeur en éditorial, à éveiller l'opinion et à lutter pour sauvegarder les intérêts de l'Église ${ }^{24}$. La direction de la revue, sise au complexe de l'Immaculée Conception, est assurée par les pères Antonio Poulin, s.j., directeur et Réal Lebel s.j., sous-directeur. Cette revue, qui a pour lectrice cible la mère de famille de la petite bourgeoisie, présente la plupart du temps en page couverture des photos de très beaux enfants ou de mères entourées d'enfants (en 1952 et en 1953, huit pages couvertures sur douze numéros annuels ont comme sujet l'enfant et la mère). La revue publie de nombreux articles sur l'éducation, mais semble peu s'intéresser à la culture et à la littérature, surtout au début des années cinquante, comme si ces domaines ne faisaient pas partie des préoccupations de son lectorat. Par contre, le cinéma, la radio et, plus tard, la télévision retiennent l'attention de l'équipe éditoriale et font l'objet non seulement d'articles mais de chroniques mensuelles. À partir du milieu des années cinquante, la télévision prend une place importante dans les préoccupations des divers rédacteurs (par exemple, le numéro de mai 1955 est entièrement consacré à ce nouveau média). Les articles, dont les sujets sont essentiellement religieux et sociaux (vie familiale), sont signés en majorité par des religieux de la Compagnie de Jésus. Les noms le plus sou- 
vent relevés sont ceux d'Alexandre Dugré, d'Iréné Beaubien, d'Adrien Théoret, de Jules Paquin et de Réal Lebel. Seules certaines chroniques spécialisées, comme la chronique médicale, sont signées par des laïcs.

Tous les mois, le père Alexandre Dugré publie une historiette édifiante sur un sujet de société comme l'alcoolisme ou la rareté des logements pour les familles nombreuses. Écrites dans une langue populaire, ces histoires présentent une vision primaire d'un comportement social et politique où seule une pensée morale catholique élémentaire est considérée comme constructive et acceptable. De plus, un grand nombre de ces historiettes ou faits divers imaginés portent sur le cheval de bataille de l'auteur: la place de la femme dans la société. Dans ses histoires, il la remet à sa seule vraie place, au foyer, car la mère dans la famille catholique, selon lui, atteint le summum de l'épanouissement et du bonheur à la maison en s'occupant de ses nombreux enfants.

Dans le domaine du livre et de la lecture, la revue ne publie pas de chronique sur les livres. Par contre, les articles sur la lecture, les bibliothèques, la littérature jeunesse, le livre et les jeunes ainsi que sur l'importance de la lecture sont nombreux, ce qui démontre l'ampleur de la préoccupation. La littérature jeunesse tient le haut du pavé avec notamment plusieurs articles de Rex Desmarchais et de Béatrice Clément sur la lecture et les jeunes, l'importance de la bibliothèque familiale comme soutien à l'éducation. En 1956, apparaît une chronique intitulée "Le courrier des lecteurs » tenue par un nouveau collaborateur, Pierre Aubry. En février, la revue annonce cette nouvelle rubrique en disant que

depuis quelques mois Ma paroisse reçoit des demandes de renseignements au sujet des lectures. [...] Nous publions ci-après quelques-unes des consultations que notre nouveau collaborateur, Pierre Aubry, a données 
à ceux et celles qui tenaient à se renseigner sur certains livres à lire ou à proscrire ${ }^{25}$.

Les questions posées sont de tous ordres (Que puis-je offrir comme lecture à ma vieille tante ? à ma jeune nièce ? ou au vicaire de ma paroisse? Ou encore, je suis bibliothécaire d'un hôpital, suggérez-moi des achats de livres distrayants pour les malades). Une grande partie des questions sont cependant de l'ordre du permis ou de l'interdit et viennent de parents désireux de contrôler les lectures de leurs enfants, surtout de leurs adolescents : "J'ai un fils de dix-sept ans qui raffole des "detectives stories" américains [...] croyez vous que la lecture de ces romans policiers lui soit dommageable $?^{26}$ » écrit une lectrice. Une autre lectrice tout aussi troublée demande : "Ma jeune fille me donne des inquiétudes depuis quelques temps elle ne s'intéresse qu'à la lecture de romans sentimentaux. [...] votre opinion s'il vous plaît ? ${ }^{27}$ "Une mère de famille d'Outremont demande : «Ne pourriez-vous pas écrire une série d'articles sur ce que nos jeunes garçons et jeunes filles peuvent lire sans danger $?^{28}$ »

Dans ses réponses, le chroniqueur répartit sa clientèle en lecteurs jeunes, lecteurs adultes et lecteurs adultes dont le jugement est formé. Cette dernière catégorie comprend plutôt des hommes, essentiellement des religieux ou des personnes appartenant à la classe des professions libérales qui ont été formées dans les collèges classiques. Par exemple, à une lectrice qui demande si le livre L'abbaye d'Evolayne de Paule Régnier est un bon livre, Pierre Aubry répond « que ce n'est pas un livre à mettre entre toutes les mains. Si vous avez plus de vingt-cinq ans, une certaine maturité d'esprit, un bon jugement, un sens moral très sûr, il vous est loisible d'en faire la lecture sans inconvénient ${ }^{29}$ ". À un jeune religieux éducateur qui lui demande ce qu'il faut penser de l'écrivain américain Erskine Caldwell, il écrit : «Le romancier américain Erskine 
Caldwell [...] dont vous me demandez "la cote de moralité" est un écrivain réaliste à fortes tendances communistes et foncièrement immoral. Très peu de ses ouvrages échappent à la censure ecclésiastique. ${ }^{30}$ ”

Il proscrit totalement de nombreux genres, auteurs et livres, tels les romans policiers américains, notamment ceux publiés par les Presses de la cité. Évidemment, des auteurs comme Colette, Zola, Gide et Françoise Sagan ne doivent absolument pas être lus, tout comme Les Provinciales de Pascal. Par contre, les collections "Le limier » et «Le masque " de chez Albin Michel sont conseillées. Pour les jeunes filles, tout comme dans les recensions du Messager qui également font beaucoup de publicité à cette collection, la collection des «Heures bleues» de La Bonne Presse est hautement recommandée.

Cette chronique sur la lecture, qui ne recense pas les livres, ne les critique pas et ne leur accorde aucune cote, fait preuve d'un pragmatisme parfaitement ignatien qui consiste à adapter le message aux personnes et aux circonstances. En laissant aux lecteurs de $\mathrm{Ma}$ paroisse, essentiellement des mères de famille, le soin de se questionner sur les ouvrages qu'elles doivent lire ou donner à lire, le chroniqueur peut gérer, jusqu'à un certain point, les bibliothèques familiales. Les directeurs de la revue préfèrent ne pas présenter à la lectrice de la revue, la mère de famille qui n'est pas toujours issue des collèges classiques, une rubrique qu'elle pourrait ne pas lire parce qu'elle serait trop loin de ses préoccupations. Ils lui laissent plutôt le soin de poser à un chroniqueur qui lui inspire de la confiance les questions qui la troublent sur les livres, les auteurs et surtout sur le rapport complexe des enfants et des adolescents face à la lecture. 
Les revues couvrant le domaine social et l'éducation

\section{Relations}

Durant la guerre, l'«École sociale populaire», qui veut contribuer à l'équilibre de la justice et de la charité entre les divers éléments sociaux et rechercher l'harmonie dans les relations humaines dans la cité, fait paraître en janvier 1941 une revue mensuelle qui a encore aujourd'hui pour nom Relations. Succédant à l'Ordre nouveau (1936-1940), le magazine Relations a, selon ses premiers éditoriaux et sa mention d'édition, l'«École sociale populaire ", une vocation essentiellement sociale. De format $8,5 \times 11$ pouces, sans aucune illustration ni photographie, Relations se caractérise par un sens très poussé de l'efficacité. Sa vocation est d'occuper " une place à part dans [l]arsenal de propagande ${ }^{31} »$ de la Société de Jésus au Québec :

[C]onsacrée à répandre la doctrine de l'Église à propos des questions d'actualité, cette revue s'est classée au premier rang des publications d'idées au Canada français; le nombre de ses abonnés et son tirage, remarquablement élevé pour une lecture aussi sérieuse et austère parfois, montrent à l'évidence la popularité de la revue et son influence pour le bien. Elle est plus qu'une simple publication : une véritable cuvre d'action religieuse et sociale ${ }^{32}$.

Dans un chapitre de leur Histoire du catbolicisme québécois consacré aux communautés religieuses, Jean Hamelin et Nicole Gagnon écrivent :

[Les Jésuites] sont perçus comme des soldats disciplinés, maîtres dans l'art militaire : disposition à utiliser le camouflage et les armes de l'adversaire, habileté à concentrer les efforts sur des points vitaux, comme la for- 
mation d'une élite, et à maîtriser la propagande par le contrôle d'institutions spécialisées à cette fin ${ }^{33}$.

Cette description peut s'appliquer à Relation, qui se veut le magazine de l'élite québécoise. En effet, la facture de la revue, sa forme austère sans illustrations ni photographies, son accessibilité grâce à un graphisme et une langue journalistique efficaces et à des chroniques qui touchent de nombreux aspects de la société peuvent être associés à la fonction de " soldats disciplinés, maîtres dans l'art militaire » qu'on accorde aux Jésuites depuis la fondation de la Société de Jésus. Les "dispositions au camouflage» ainsi que l'utilisation des " armes de l'adversaire » correspondent à la diversité des rubriques qui couvrent l'ensemble de la vie urbaine tant au niveau social que culturel, telles les "Chronique cinéma", "Chronique littéraire ", "Chronique théâtre », recensions des nouveautés littéraires avec "Livres récents", ainsi qu'aux aperçus de la politique internationale avec « Horizon international» et de la vie sociale dans "Avec ou sans commentaires ». Les titres de ces rubriques démontrent que les éditeurs de Relations abordent des sujets considérés comme délicats par le monde religieux.

Dès son premier numéro, la revue publie sous le titre "Livres récents » des recensions d'ouvrages nouvellement sur le marché. Pierre Angers, s.j., dans son livre Foi et littérature, traduit bien la pensée des recenseurs de la «Maison des écrivains » en écrivant que :

[L]a diffusion du livre, accrue par de puissants moyens, n'est pas à blâmer pour elle-même ; ni la vente d'ouvrages de mauvais maîtres, dont la pensée féconde à plusieurs égards, doit rester accessible à ceux qui, par leurs fonctions ou pour tout autre motif valide, ont intérêt à la connaître et à l'étudier. [...] La critique qui recommanderait ces œuvres en soulignant les mérites qui leur 
sont propres, mais sans formuler les réserves qui s'imposent, la critique qui les exalterait avec une admiration sans borne ne s'inspire plus des vues de la foi et expose la conscience des catholiques à de graves mépri$\operatorname{ses}^{34}$.

Le lecteur ciblé par la chronique appartient à la catégorie de lecteurs que le chroniqueur de Ma paroisse Pierre Aubry nomme "lecteur adulte dont le jugement est formé ", c'est-àdire les intellectuels, les professionnels et les religieux qui ont fréquenté les collèges de la Société de Jésus ou d'autres communautés.

La lecture de ces recensions permet de constater qu'elles sont largement positives. Cependant, les recenseurs tiennent compte en priorité de la valeur morale, sociale et religieuse de l'ouvrage avant de considérer sa valeur strictement littéraire. Ainsi, René Girard s.j., au sujet du livre de Françoise Gaudet-Smet intitulé Heures d'amour, publié par les Éditions Fides, écrit que : "[T] out ce qui sert la famille, aujourd'hui, doit être exploité à fond. Or, ce livre est un excellent instrument d'éducation familiale. Il mérite donc une large diffu$\operatorname{sion}^{35}$ ». Le critère moral domine tous les autres, même quand le recenseur sait qu'il est face à un chef-d'œuvre littéraire. Quand Robert Morency présente Le diable au corps de Radiguet comme un « roman d'une réelle valeur littéraire, [il ajoute immédiatement] mais franchement immoral ${ }^{36}$ ", il poursuit en disant : " []] faut reconnaître que l'auteur a du style. Mais la critique intégrale doit déborder les considérations proprement littéraires. Or, le roman de Raymond Radiguet est immoral ${ }^{37}$. " L'éditeur, Bernard Valiquette, subit tout comme l'auteur les foudres du recenseur qui termine en écrivant «le roman de Radiguet nous paraît nocif. Aussi regrettons-nous à bon droit qu'une maison d'édition de chez nous réédite un tel ouvrage ${ }^{38}$. » 
Parmi les auteurs bien cotés par les recensions de « Livres récents » figurent des jésuites, notamment François Hertel et Pierre Angers. Les auteurs publiés par les Éditions de l'Arbre $^{39}$, Anne Hébert, Raïssa Maritain, Roger Lemelin, JeanJules Richard et Robert Charbonneau sont positivement cotés. Neuf jours de baine, le premier roman de Richard, a une réception critique positive, sauf de la part de certains religieux qui dans Lectures ${ }^{40}$ ou La Revue dominicaine ${ }^{41}$ stigmatisent l'anticléricalisme de l'auteur. Dans Relations, le jésuite Paul-Émile Racicot écrit de Jean-Jules Richard que :

[sa] sensibilité reste saine et juste, dans la haine comme dans l'amour [et que...] l'auteur manifeste une rare capacité d'introspection et d'idéalisme ; il présente des images abondantes et suggestives, il possède aussi une gamme très riche de sentiments. Ce n'est pas un mince éloge de pouvoir dire à l'auteur d'un premier livre que les ressources spirituelles dépassent de beaucoup la maitrise de son métier ${ }^{42}$.

Cette critique très positive s'adresse à l'œuvre de Richard bien sûr, mais également aux Éditions de l'Arbre qui la publient. Bien différente est la réception du Feu dans l'amiante, publié à compte d'auteur par Jean-Jules Richard en 1956, et que la revue qualifie de «roman triste et triste roman ${ }^{43}$ ». Roger Lemelin, quand il publie $A u$ pied de la pente douce à l'Arbre, a une recension positive; Jacques Tremblay écrit que «[...] ce roman atteint [...] par la signification profonde de ses peintures et de ses réflexions, par la qualité des images, par l'agencement de ses foyers d'intérêt, par la stylisation de ses caricatures, par la finesse aiguë de l'écriture, à l'universalité du grand $\operatorname{roman}^{44}$ ». Mais quand, en février 1949, «Livres récents » recense Les Plouffe, publié aux Éditions Bélisle à Québec, Ernest Gagnon du Collège Sainte-Marie écrit que " [...] ce livre est un faux pas. Une attitude plus humble et plus sincère devant la vie est primordiale chez un romancier [et que] ce roman ne 
fera pas le tour du monde ${ }^{45}$ ». Un an plus tard, Ernest Gagnon, au sujet de Fantaisie sur les péchés capitaux, que Lemelin publie chez Beauchemin, révise un peu son jugement : « [L]'auteur a donné la preuve qu'il peut se renouveler, incarner un sujet et l'exprimer dans une langue littéraire. Ce redressement peut le sauver ${ }^{46} . »$

La littérature québécoise est bien mise en valeur dans "Livres récents », surtout durant la période 1940-1950. Si nous avons rencontré certaines restrictions au sujet des ouvres de Roger Lemelin, leur lecture n'est pas interdite. Pour ce qui est de Ringuet, la recension de Trente arpents et Le poids du jour, signée Jacques Tremblay, est positive et élogieuse, mais PaulÉmile Racicot, qui recense Fausse monnaie en 1948, écrit que «Ringuet n'a laissé parler que le styliste, le lecteur attend toujours l'homme. Cette brillante monnaie ne sonne pas assez juste à l'oreille canadienne ${ }^{47}$. » En 1956, la biographie de Félix Leclerc, Moi mes souliers, est présentée avec enthousiasme par André Surprenant qui trouve qu'elle « charme par son accent personnel, par son humour sain et constant ${ }^{48}$ ».

Les œuvres étrangères ou féminines sont plus sévèrement critiquées et sont parfois sujettes à l'anathème. La raillerie est utilisée avec plaisir quand l'auteur est une femme. Ainsi, à propos d'un roman intitulé L'espion de l'âle-aux-Coudres, de Lætitia Filion, le recenseur dit qu'il «n'enrichit pas notre littérature ». Au sujet d'un roman intitulé Plympton house, écrit par Paule d'Oncin, Rodolphe Dubé, alias François Hertel, dit que son «ami Valiquette eût mérité davantage des lettres canadiennes et des lettres françaises en ne publiant pas cet ouvrage ${ }^{49}$ ». À propos d'Isabelle Tonarelli, l'auteur de Jeunes femmes, Georges-Henri d'Auteuil écrit qu'elle est « une femme, une licenciée ès lettres, une diplômée d'études de philosophie (vous voyez le genre!) ${ }^{50}$ » et poursuit en déplorant « le ton de l'ouvrage d'un cynisme et d'une crudité de langage 
tout à fait déplacés ${ }^{51}$ ». La rare interdiction que nous ayons relevée concerne un roman signé Thyra Seillière, Lïntelligence $d u$ caur, au sujet duquel le recenseur, E. Forget, déclare que " [l]'Église défend d'acheter, de lire, de conserver, de prêter un tel livre; il faut même le brûler ${ }^{52}$ ».

Des auteurs comme Gide et Proust, quand ils sont mentionnés, et ils ne le sont pratiquement jamais, sont considérés comme des suppôts de Satan qui ont perverti la jeunesse européenne, française particulièrement. La méfiance engendrée par leur signature se fait sentir même si l'ouvrage est tout à fait anodin. Une recension, signée René Latourelle, signale la sortie aux Éditions Jacques Shiffrin, à New York, de Interviews imaginaires d'André Gide. Le recenseur, tout en soulignant que «l'ensemble est inoffensif» et que «[d]u style, il faut admirer le dépouillement, la simplicité linéair ${ }^{53}$ ", formule cependant des réserves à cause de "la forme dialoguée adoptée par l'auteur [qui] favorise les déguisements de la pensée, les reprises, les compromis ${ }^{54} \cdots$.

Une des recensions les plus négatives publiées par Relations est celle qui signale la réédition au Québec, chez Valiquette, de Jean Christophe et, chez Variétés, de Vie de Beethoven, de Romain Rolland. Le recenseur, Paul Bélinau, écrit à ce propos que «les éditeurs canadiens de Romain Rolland ont leurs raisons [mais que néanmoins son œuvre est] œuvre de régression sans autorité et de valeur null ${ }^{55}$ \%.

Dans Relations, les Jésuites préfèrent dans l'ensemble ne pas utiliser l'arme de l'interdit. Ils portent plutôt sur les œuvres et sur leurs auteurs un jugement catholique destiné à informer et éduquer le lecteur. Ce choix correspond à une stratégie qui fait appel à la pédagogie. En ce sens, la chronique «Livres récents », qui préfère convaincre qu'interdire, est une arme, l'arme non violente de la persuasion. Il faut cependant tenir compte du fait que le lectorat de Relations, parce 
qu'il est constitué majoritairement de membres de l'élite formée à la pédagogie ignatienne, est considéré par les rédacteurs de la revue comme aptes à comprendre leurs jugements et à les suivre, s'ils ne les précèdent pas.

\section{College et famille}

Collège et famille voit le jour en janvier 1944, publié par «Les collèges de la Compagnie de Jésus » au Canada et produit par l'Imprimerie du Messager. Le contenu éditorial de la revue, principalement axé sur l'éducation, s'adresse aux parents des élèves de ces collèges. Outre son rôle de liaison, College et famille est une source d'informations pour ceux-ci, qui devraient être, en retour, des lecteurs actifs, voire des rédacteurs occasionnels ${ }^{56}$. Cette collaboration suppose la surveillance de l'enfant comme du jeune homme et un échange maitres-parents pour le plus grand bien du collégien.

La revue publie toutes sortes d'articles destinés aux parents, portant sur des sujets variés en rapport avec l'enfant et l'adolescent. La lecture, le livre et la littérature font partie des sujets que la revue favorise. La chronique de recensions «Des livres » fait partie de la volonté de protection de la jeunesse préconisée par la communauté comme l'indique en 1948 la toute première chronique "Des livres » qui commence par ces mots :

Chaque livraison vous apportera désormais une couple de recensions d'ouvrages qui se rapportent de quelque manière à l'éducation. Qu'on n'y cherche nulle réclame, mais uniquement le souci de fournir des jugements personnels sur des livres utiles, nécessaires ou discutables $^{57}$

Le nombre de recensions que Collège et famille publie atteste l'importance considérable que la communauté accorde à la lecture en éducation. Outre la chronique "Des livres", 
plusieurs articles destinés à orienter les lectures familiales et à compléter la mission pédagogique de la revue auprès des parents abordent des sujets tels que la lecture et la culture, la lecture et les enfants, la littérature et la morale, le livre et sa diffusion $^{58}$.

Dans leur ensemble, les articles critiques de College et famille jugent les œuvres comme "bonnes" ou " mauvaises " non pas en fonction de leurs qualités littéraires, mais de la vie privée des auteurs ou de la perception que les religieux ont de leur réputation. Dans « La critique littéraire des Jésuites », nous mentionnons un article portant sur la biographie de Mermoz par Joseph Kessel qui dit que : incroyant et athée, à l'instar du sujet de son ouvrage, Kessel ne peut qu'écrire un livre " mauvais", sur le plan moral bien entendu ${ }^{59}$.

Certains de ces articles engendrent une profonde méfiance vis-à-vis de la chose littéraire, et la littérature reste suspecte pour certains religieux et ne peut être rachetée que par l'impeccable orthodoxie des auteurs. Même la lecture des œuvres d'un catholique comme Mauriac, que l'ensemble de la critique considère comme un écrivain de talent, devient impropre à de jeunes esprits, car l'auteur a la réputation d'avoir une personnalité déséquilibrée à cause de son trop grand penchant pour l'introspection. Par contre Péguy et surtout Claudel, dont l'histoire personnelle est exemplaire (à dix-huit ans il découvre la foi catholique et la littérature), sont perçus comme des modèles enrichissants pour de jeunes personnalités en construction. Ces articles, qui servent avant tout à transmettre l'idéologie conservatrice que certains jésuites canadiens, à l'instar de la hiérarchie religieuse du pays, tentent de préserver après la guerre, ne peuvent donc pas être qualifiés de critiques. Ce sont des articles à teneur essentiellement pédagogique. 
Cependant à la fin des années 1950, quelques jeunes représentants de la communauté opposent aux propos manichéens de leurs aînés des idées plus libérales qui tiennent compte de l'évolution incontestable de la pensée de la Société. Dans son étude de l'œuvre de Claudel, André Vachon, s.j. propose une approche de la littérature plus conforme à son contexte social et, dans une moindre mesure, Pierre Angers, qui a toujours ressenti le besoin de différencier la critique littéraire et le jugement moral, représente aussi un courant plus progressiste parmi les auteurs de cette revue ${ }^{60}$.

Il est intéressant de remarquer que les stratégies établies par l'ensemble des communautés religieuses du Québec ne pourront pas arrêter les avancées idéologiques et sociétales qui se dessinent à la fin de la guerre et que la Révolution tranquille mettra de l'avant au cours des années soixante. Il est également remarquable de voir que les enseignants jésuites, dès le milieu des années cinquante, réviseront leur stratégie pédagogique, comme l'écrit Guy Bourgault en 1955 dans un article intitulé "Claudel et la vocation de l'homme", pour "permettre à l'adolescent de prendre conscience du monde qui l'entoure, pour y occuper sa vraie place ${ }^{61} »$. Ce tournant qui semble être négocié par une génération de religieux plus jeunes peut nous permettre d'envisager que le rapport des Jésuites à la lecture et aux cuvres littéraires évolue vers une nouvelle compréhension qu'ils entendent faire partager aux jeunes qu'ils forment.

\section{$* * *$}

L'Apostolat de la prière, à l'origine des œuvres de la Société de Jésus, a permis à cette communauté de se forger des outils aussi pertinents et originaux que diversifiés pour atteindre ses buts : étendre son influence intellectuelle sur une société. L'ensemble socioculturel de l'Immaculée Conception, 
avec l'Imprimerie du Messager, la Maison des écrivains, la direction de l'«École sociale populaire», étend un maillage serré sur la société qui permet aux religieux d'exercer une action structurante sur la lecture, le livre, et par extension, la littérature. Grâce à l'imprimerie du Messager, la Société de Jésus produit les instruments essentiels à sa propagande, ses divers organes de presse étendent son influence sur divers secteurs sociaux. Le complexe éditorial de l'Immaculée Conception sert essentiellement à la publication de leurs nombreux tracts, revues et brochures destinés à soutenir la pensée jésuite. Cette organisation éditoriale permet à la communauté de structurer la pensée de l'ensemble des lecteurs dans le sens déterminé par les institutions cléricales.

Comme le mentionne Roland Barthes dans Sade, Fourier, Loyola ${ }^{62}$ et comme l'attestent les auteurs jésuites dans plusieurs écrits importants, une des clés de la culture, selon la Société de Jésus, est la lecture des œuvres littéraires, avec la censure comme corollaire. C'est pourquoi, au Québec, au cours des années trente à soixante, la Société de Jésus diffuse plusieurs ouvrages critiques signés par des religieux tant québécois que français, mais surtout elle publie de très nombreux articles et recensions dans ses diverses revues. Cette stratégie est destinée à encadrer le lecteur pour qu'il s'approche de l'œuvre littéraire avec précaution ainsi que le préconise $\mathrm{Al}$ phonse de Parvillez, s.j., dans Le livre au service du Christ ${ }^{6.3}$. Dans le même ordre d'idées, François Charmot, dans La pédagogie des Jésuites, écrit qu'un enfant: " ne se forme pas loin de tout mal, mais dans la lutte contre le mal. La sagesse veut qu'on proportionne la tentation à ses forces, mais non qu'on supprime le combat ${ }^{64} »$. Manifestement, ces préceptes guident l'évaluation de la production littéraire de la Compagnie de Jésus au Québec et les actions qu'elle entreprend pour en contrôler la diffusion. D'autres composantes comme le cinéma et surtout la télévision viennent changer la donne et obligent la 
communauté aux alentours des années cinquante à créer des rubriques idoines pour surveiller ces nouveaux médias.

La spiritualité de la Compagnie de Jésus oriente les propos des auteurs de la communauté et, par voie de conséquence, influence les lecteurs. Cette spiritualité est le fondement d'une argumentation que les Jésuites utilisent pour convaincre. Des mots comme discernement (disposition de l'esprit à juger clairement et sainement les choses, voir Le Robert), perception (dans le sens de représentation intellectuelle), volonté (disposition à vouloir et à agir), humanisme (dans son sens chrétien, c'est-à-dire épanouissement de la personne humaine en Dieu), typiques de la dialectique jésuite, se retrouvent toujours dans les diverses publications ignatiennes.

Les préoccupations de la presse jésuite rejoignent celles de la presse catholique en général. Morale, famille et problèmes sociaux y sont des thèmes récurrents. Les enseignements de l'Église et son histoire sont présents au même titre que l'histoire de la communauté et la médiatisation de ses apostolats. Les articles consacrés à la diffusion de l'imprimé et à la lecture reviennent à intervalles réguliers dans chacune des revues dépouillées. La stratégie des Jésuites entend conditionner et influencer le lecteur afin qu'il s'autocensure. Cette forme d'accompagnement, vraiment importante au cours des années trente, se transforme légèrement durant les décennies suivantes car d'autres composantes, notamment la guerre, obligent la communauté à réviser ses tactiques et à se mettre en harmonie avec les nouvelles circonstances, selon un des principes importants de la spiritualité ignatienne, le principe d'adaptation.

Les membres de la Domus scriptorum qui signent majoritairement les chroniques ou les articles sur la lecture et la littérature jugent et évaluent d'un point de vue uniquement moral la production littéraire essentiellement francophone 
accessible au Québec. Les diverses chroniques de recensions des revues jésuites ne sont certainement pas des chroniques de critique littéraire, leur but étant surtout d'inculquer au lecteur la méfiance vis-à-vis de la chose écrite, de le mettre en garde contre les "mauvais livres " et de lui apprendre à s'autocensurer. Cependant, parce que les Jésuites sont des intellectuels et des enseignants, les chroniques "livres » veulent promouvoir la lecture, une lecture saine et adaptée aux diverses couches de la population.

\section{NOTES}

' Jean Hamelin et Nicole Gagnon, Histoire du catholicisme québécois. Le XX' siècle, vol. 1, 1898-1940, Montréal, Boréal express, 1984, p. 139.

2 Jean Lacouture, Jésuites, vol. 2, Les revenants, Paris, Seuil, 1991, p. 404.

${ }^{3}$ Alexandre Dugré, « La presse catholique », Le Messager canadien, sept. 1935, p. 381.

${ }^{4}$ La direction des archives des Jésuites à Saint-Jérôme, que j’ai contactée dès le début de mon travail sur la société de Jésus, n'a fourni que des fins de non recevoir à chacune de mes demandes. Au sujet du Scolasticat de l'Immaculée Conception, de la Maison des écrivains et de l'Imprimerie du Messager par exemple, on m'a répondu que les archives étaient en traitement et qu'elles ne pourraient être consultées par les chercheurs avant plusieurs années. Je dois dire par contre que les services d'archives du collège Brébeuf, du centre Vimont et du Gésu m'ont tous fourni, dans la mesure de leurs moyens, les renseignements que je leur demandais. De plus, les pères Louis-Bertrand Raymond et Pierre Angers, que j'ai rencontrés longuement, ont été d'une grande compréhension et d'une générosité extraordinaire.

${ }^{5}$ Voir Jacques Michon, dir., Histoire de l'Édition littéraire au Québec au XX" siècle, vol.1, La naissance de l'éditeur, 1900-1939, Saint-Laurent, Fides, 1999, p. 351.

"Simone Vannucci, « Rôle structurant des actions de la Compagnie de Jésus sur la littérature et l'édition au Québec, 1930-1960", thèse de doctorat (lettres et communications), Université de Sherbrooke, 2003, p. 105.

" "La maison Bellarmin, un centre d'action religieux et social ", Ma paroisse, janv.1950, p. 10. 
${ }^{8}$ Maparoisse, janv. 1950, p. 9.

"Ibid.

${ }^{10} \mathrm{Ibid}$.

${ }^{11}$ Parmi les caractéristiques du modèle culturel ignatien, on relève notamment le souci d'efficacité, l'emphase mise sur le travail intellectuel et le contrôle de l'imagination qui font du jésuite un contemplatif dans l'action, qui possède l'intelligence de l'autre et du temps.

${ }^{12}$ Pierre Matte, «La bibliothèque de la maison Bellarmin », dans Georges-A. Chartrand, dir., Livre, bibliothèque et culture québécoise. Mélanges offerts à Edmond Desrochers, s.j., Montréal, ASTED, 1977, t. I, p. 63.

${ }^{13}$ Le Messager canadien, juil.-août 1954, p. 549.

${ }^{14} \mathrm{~L}$ '« École sociale populaire » est l'aboutissement « d'une idée longuement mûrie par le jésuite Léonidas Hudon » et est, selon la même source, "un calque d'un mouvement français, l'Association catholique de la jeunesse française ». En 1910 déjà, Pierre Gerlier, président de l'Association de la jeunesse catholique française, et Thellier de Poncheville, de passage à Montréal à l'occasion du congrès eucharistique, encouragent les Ligues du Sacré-Cœur à s'engager sur le terrain de l'organisation professionnelle et de la vulgarisation de la doctrine sociale. Voir Hamelin et Gagnon, op. cit., p. 227.

${ }^{15}$ Ibid, p. 228

${ }^{16}$ C'est à la fin des années vingt, quand le père Joseph-Papin Archambault prend en main les destinées de l'« École sociale populaire » et en devient la tête pensante et le moteur, que cet organisme prend son envol. Nommé directeur de la « Maison des écrivains » au début des années trente, il reste administrateur de divers organismes. De plus, il s'occupe de publications qui ont leur bureau directeur au scolasticat et réunit le tout sous un même comité à l'Immaculée Conception. Voir Christiane Demers, La conception de la démocratie chez l'"École sociale populaire », 1930-1960, mémoire de maitrise (histoire), Université Laval, 1979, f. 21-24.

${ }^{17}$ G.-Raymond Laliberté, « Dix-huit ans de corporatisme militant, l”'École sociale populaire" de Montréal, 1933-1950", Recherches sociographiques, vol. XXI, n 1-2, janv.-août 1980, p. 56.

${ }^{18}$ Maparoisse, janv. 1950, p. 10.

${ }^{19}$ Laliberté, loc.cit., p. 62.

${ }^{20}$ Le Messager canadien, «Présentation Nouvelle », vol. 59, n 1, janv. 1949, p. 1. 
21 Voir Dominique Avon et Philippe Rocher, Les jésuites et la société française,Toulouse, Éd. Privat, 2001, pp. 67-68.

22 "Un quatrième Messager du Sacré-Caur publié au Canada », Le Messager canadien, 1956, p. 319.

${ }^{23}$ Joseph-Papin Archambault, s.j., " Les livres d'étrennes », Le Messager canadien, janv. 1930, p. 29.

${ }^{24}$ «Transformation », Ma paroisse, fév. 1945, p. 1.

${ }_{25}^{25}$ «Le courrier du lecteur », Ma paroisse, février 1956, p. 26.

${ }^{26}$ Ibid.

${ }^{27}$ Ibid.

${ }^{28}$ «Le courrier des lecteurs » Maparoisse, juin 1957, p. 19

${ }^{29}$ «Le courrier des lecteurs » Maparoisse, août 1956, p. 21.

${ }^{30}$ Ibid.

${ }^{31}$ Ma paroisse, janv. 1950, p. 10.

${ }^{32}$ Ibid.

${ }^{33}$ Hamelin et Gagnon, op. cit., p. 139.

${ }^{34}$ Pierre Angers, Foi et littérature, Montréal, Beauchemin, 1959, p. 26.

${ }^{35}$ René Girard, s.j., Relations, 1943, p. 195.

${ }^{36}$ Robert Morency, s.j., Relations, juin 1944, p. 166.

${ }^{37}$ Ibid.

${ }^{38}$ Ibid.

${ }^{39}$ Les directeurs de Éditions de l'Arbre, Claude Hurtubise et Robert Charbonneau sont d'anciens élèves du Collège Sainte-Marie.

40) Jean-Marie Gaboury, "Études critiques. Salut au Kentucky. Neuf jours de haine ", Lectures, mai 1948, pp. 201-205.

${ }^{41}$ Henri-Marie Bradet, o.p., "L'esprit des livres », La revue dominicaine, mai 1948, p. 319.

12 Paul-Émile Racicot, s.j., Relations, 1948, p. 190.

${ }^{43}$ Alexandre Dugré, s.j., Relations, 1956, p. 204.

${ }^{H+}$ Jacques Tremblay, s.j., Relations, 1944, p. 195. 
${ }^{45}$ Ernest Gagnon, s.j., Relations, 1949, p. 57.

${ }^{46}$ Ernest Gagnon, s.j., Relations, 1950, p. 55.

${ }^{47}$ Paul-Émile Racicot, s.j., Relations, 1948, p. 62.

${ }^{48}$ André Surprenant, s.j., Relations, 1956, p. 175.

${ }^{49}$ Rodolphe Dubé, s.j., Relations, 1943, p. 83.

${ }^{50}$ Georges-Henri d'Auteuil, s.j., Relations, 1943, p. 306.

${ }^{51}$ Ibid.

${ }^{52}$ E. Forget, s.j., Relations, 1948, p. 311.

${ }^{53}$ René Latourelle, s.j., Relations, 1944, p. 278.

${ }^{54}$ Ibid.

${ }^{55}$ Paul Bélinau, s.j., Relations, 1944, p. 278.

${ }^{56}$ Voir l'article du père Antonio Dragon, «Ce que le collège attend de la famille ", Collège et famille, 1944, p. 9, dans lequel il précise ce que le collège entend par collaboration familiale.

${ }^{57}$ Collège et famille, 1948, vol. V, n 1, p. 47.

${ }^{58}$ Pour une analyse des recensions de livres et des articles portant sur la lecture dans College et famille, voir Simone Vannucci, « La critique littéraire des Jésuites », dans Josée Vincent et Nathalie Watteyne, dir., Autour de la lecture. Médiations et communautés littéraires, Québec, Éditions Nota bene, 2002, pp. 131146.

${ }^{59}$ Ibid., p. 143.

${ }^{(1)}$ Lors de notre rencontre d'octobre 2002, le père Angers nous a dit qu'il a toujours pensé qu'un catholique trop intransigeant se ferme à beaucoup de choses. Mais, dit-il, «mon éducation familiale et la fréquentation de ma paroisse dominicaine de Notre-Dame de Grâce ont beaucoup aidé à ma vision d'un catholicisme plus ouvert. Cependant les commentaires sur Foi et littérature n'ont pas été toujours bons. On m'a reproché de présenter les choses sous un angle trop large. On doit, dit-il, se replacer dans le contexte de l'époque et savoir qu'aujourd'hui la conscience morale des catholiques s'est considérablement élargie » : Vannucci, op. cit., Entretien oct. 2002, annexe 2, p. 355.

${ }^{61}$ College et famille, 1955, p. 124. 
${ }^{12}$ Roland Barthes, Sade, Fourier, Loyola, Paris, Seuil, 1971, p. 45. (Coll. «Tel quel »).

${ }^{63}$ Alphonse de Parvillez, s.j., Le liure au service du Christ, Paris, Éd. Spes, 1940, $252 \mathrm{p}$.

${ }^{6+}$ François Charmot, s.j., La pédagogie des jésuites. Ses principes, son actualité, Paris, Éd. Spes, 1943, p. 510. 